\title{
Concurrence et confusion des discours sur le crime organisé en Russie
}

\section{Gilles Favarel-Garrigues}

\section{(2) OpenEdition \\ 1 Journals}

Édition électronique

URL : http://journals.openedition.org/conflits/555

DOI : $10.4000 /$ conflits.555

ISSN : $1777-5345$

\section{Éditeur :}

CCLS - Centre d'études sur les conflits lilberté et sécurité, L'Harmattan

\section{Édition imprimée}

Date de publication : 1 juin 2001

ISBN : 2-7475-1117-0

ISSN : 1157-996X

\section{Référence électronique}

Gilles Favarel-Garrigues, "Concurrence et confusion des discours sur le crime organisé en Russie », Cultures \& Conflits [En ligne], 42 | été 2001, mis en ligne le 04 décembre 2002, consulté le 30 mars 2021. URL : http://journals.openedition.org/conflits/555 ; DOI : https://doi.org/10.4000/conflits.555

Ce document a été généré automatiquement le 30 mars 2021.

Creative Commons License 


\title{
Concurrence et confusion des discours sur le crime organisé en Russie
}

\author{
Gilles Favarel-Garrigues
}

\section{NOTE DE L'AUTEUR}

Je remercie Anton Oleïnik et les lecteurs anonymes de ce texte pour leurs commentaires.

\section{Concurrence et confusion des discours sur le crime organisé en Russie}

Le crime organisé russe a connu, tout au long des années 1990, un succès remarquable dans les forums politiques et économiques russes, occidentaux et internationaux, pour des raisons partiellement connues. La fin du contexte bipolaire a non seulement conduit à renoncer à une vision du monde fondée sur une forme stable d'ordre international, mais a également provoqué l'émergence de nouvelles préoccupations, liées à l'ouverture des frontières et à la désagrégation interne de nombreux Etats postcommunistes, notamment de la Russie. La perte de l'ennemi consacré et l'émergence de nouvelles menaces liées à l'espace post-communiste ont conduit à intégrer les « mafias russes » ou « mafias rouges » dans le discours américain sur la sécurité nationale ${ }^{1}$.

L'objectif de cet article est moins de déconstruire les discours occidentaux sur la menace incarnée par les «mafias rouges » que de comprendre comment ce thème a émergé dans le contexte soviétique, puis post-soviétique, avant de se diffuser dans le monde occidental. Je souhaite montrer comment les modes et enjeux de la constitution d'un problème relatif au crime organisé en Russie ont contribué à façonner les représentations occidentales. Il s'agit notamment de préciser comment se sont 
accumulées les connaissances en Russie, à partir de quelles sources et en particulier comment un savoir à prétention scientifique a pu alimenter, confirmer ou infirmer les discours policiers, journalistiques et politiques sur ce sujet. Les recherches sur le crime organisé russe se développent depuis la fin des années 1980 : de nombreux ouvrages, thèses de doctorats, articles de revue ou colloques ont été consacrés à ce sujet. En établissant un bilan de cette littérature (1988-2001), l'objectif n'est pas de juger les recherches menées, mais de comprendre comment a émergé un savoir spécialisé relatif au problème du crime organisé.

Le terme de «problème » est employé en référence aux travaux de Murray Edelman : il s'agit d'une situation préoccupante qui constitue un enjeu politique du fait des " différences existant entre ses définitions ", de "l'hétérogénéité des intérêts propres aux différents groupes qui s'efforcent chacun de faire adopter des lignes d'action présentées comme des solutions " ${ }^{2}$. Un problème "focalise l'attention sur un nom désignant une situation indésirable ou une menace pesant sur le bien-être. Les activités gouvernementales qui sont rationalisées par cette focalisation consistent en une séquence d'allégations et d'actions ambiguës qui varient au gré des circonstances et sont souvent mutuellement incompatibles du fait des réponses distinctes qu'elles apportent aux différents groupes d'intérêts en compétition $»^{3}$. Dans l'introduction de l'édition française de l'un de ses ouvrages paru en 1991, Murray Edelman pressent que ses thèses, qui concernent initialement les "pays démocratiques industriellement avancés ", sont susceptibles de présenter un intérêt heuristique pour l'étude des pays post-communistes, dans la mesure où la construction des problèmes sociaux dépend notamment du développement des médias et de la généralisation de l'accès aux informations politiques ${ }^{4}$.

La perspective que je souhaite confronter aux discours qui perçoivent le crime organisé comme un fait social objectivé ne doit pas conduire à un relativisme outrancier. La volonté de comprendre les conditions de production des discours et des actes politiques, policiers et judiciaires relatifs à cette forme de délinquance, ne peut en aucun cas conduire à nier l'existence ou la gravité des pratiques hétérogènes incluses dans les différentes définitions du "crime organisé $»^{5}$. Cependant, l'emploi de l'expression de «crime organisé » ne revient pas, dans ma perspective, à la valider et à considérer qu'elle désigne un phénomène social indiscutablement constitué ${ }^{6}$, mais simplement à admettre qu'une littérature, des discours, des budgets et des services répressifs spécialisés lui sont explicitement associés. Enfin, l'importance que j'accorde aux discours dans le cadre d'un article consacré à l'analyse du savoir sur le crime organisé russe ne signifie pas un désintérêt pour leur éventuelle conversion en pratiques ${ }^{7}$.

La criminalité organisée est en Russie un problème constitué récemment, à partir de sources rares et selon des références cognitives troublées par la concurrence et la confusion des significations associées au terme de mafia dans le contexte soviétique. Dès la fin des années 1980, le problème de la criminalité organisée, ou problème mafieux, devient un enjeu politique important dans les affrontements opposant «conservateurs » et «réformateurs». La définition et l'explication de ce problème varient alors en fonction de la manière dont est envisagé le développement souhaitable $\mathrm{du}$ pays. Les formulations concurrentes s'appuient chacune sur un corps de connaissances composé de discours politiques, journalistiques et scientifiques qui se valident mutuellement. Ces formulations se diffusent à l'étranger, en particulier aux Etats-Unis, où elles étayent notamment des positions idéologiques relatives à la 
politique menée à l'égard de la Russie. L'homogénéité des discours sur la criminalité organisée, formulés par des coalitions d'acteurs à partir de considérations idéologiques, se maintient durant les années 1990, mais leur contenu varie et les travaux scientifiques tendent progressivement à se distinguer.

\section{Le triple discours soviétique sur la criminalité organisée}

Contrairement à une idée répandue, le discours des dirigeants politiques soviétiques dans les années 1970 et 1980 sur la criminalité en général et sur le crime organisé en particulier ne se réduisait pas à une position officielle invariable. Il existait en fait un répertoire de discours associés à ce thème. Paradoxalement, alors que le crime ne représentait pas encore un problème social, c'est-à-dire un enjeu politique opposant différents groupes autour de sa définition et de sa résolution, il suscitait déjà des usages politiques différenciés. Monopolisant la formulation du problème criminel, les dirigeants soviétiques maniaient, simultanément ou en alternance, trois discours. Le premier reflétait une vision idéalisée de l'accomplissement du programme marxisteléniniste, qui annonçait l'éradication du crime par la suppression de ses causes sociales ${ }^{8}$. Dans la Grande Encyclopédie Soviétiquede 1975, l'entrée « criminalité » donne accès à un discours emblématique :

«Dans la société socialiste, les causes fondamentales de la criminalité ont été anéanties. [...] La criminalité n'est pas encore totalement éradiquée car le socialisme, dans ses relations économiques et morales, ne s'est pas encore débarrassé de toutes les 'taches de naissance' de l'ancienne société. Les causes de la criminalité sont les séquelles du passé qui se manifestent encore dans différents secteurs de la vie sociale, mais aussi dans la conscience, la psychologie et la vie quotidienne des individus. Ces causes [...] sont organiquement étrangères à la société socialiste; au sein de celle-ci, la criminalité elle-même constitue une régression. [...] En URSS, le niveau de la criminalité est trois à quatre fois inférieur à ce qu'il était dans les années 1920-1930 et la criminalité professionnelle a été éradiquée ${ }^{9}$.

L'expression de "criminalité professionnelle» désignait alors la pègre, le milieu criminel. Elle était fréquemment employée au cours des années 1970-1980 à propos des professionnels du vol, du jeu, de la prostitution, avec leurs traditions et coutumes (rites d'intronisation, signes extérieurs d'appartenance, culture carcérale), en somme à propos de ceux qu'à partir de la fin des années 1980 on désignera comme membres du «crime organisé » ${ }^{10}$. L'« éradication de la criminalité professionnelle» justifiait l'absence de travaux criminologiques sur cette question. Il est possible que, conformément à ce qu'affirment aujourd'hui plusieurs chercheurs issus des instituts de recherche scientifique du ministère de l'Intérieur ou de la Prokouratoura générale de la Fédération de Russie, une littérature grise ait existé sur ce sujet dès les années 1970, mais aucune preuve ne le confirme ${ }^{11}$. En 1989, un chercheur de l'Institut de la Prokouratoura déclare : «Il est difficile de croire qu'il y a à peine quatre ou cinq ans, il nous était refusé d'intégrer dans le plan d'Etat des activités de recherche scientifique des objectifs relatifs à l'étude de la criminalité organisée. Par la suite, des objectifs en termes d'études des groupes criminels organisés ont été acceptés. Ce n'est qu'en 1988 que l'expression 'criminalité organisée' est apparue dans le plan $»^{12}$. 
Ce discours officiel niant l'existence de la pègre était formulé dans un contexte où sa persistance était néanmoins certaine. Au cours des années 1960, de nombreux films comiques populaires montraient les démêlées d'un "cave " pénétrant fortuitement dans le monde de la pègre ${ }^{13}$. Les chansons du milieu criminel étaient bien connues. Même censurés, de nombreux ouvrages décrivaient les pratiques en vigueur dans le "monde des voleurs", que ce soit dans le cadre d'ouvrages sur le milieu carcéral ${ }^{14}$ ou sur la société soviétique ${ }^{15}$. Lors des recherches que j'ai menées à partir des archives policières et judiciaires de la région de Sverdlovsk, j'ai pu relever plusieurs traces de l'existence de la pègre. Les archives mentionnent dès les années 1960 l'existence de bandes de criminels détournant du bois vers les régions du Sud de l'Union soviétique avec la complicité d'agents économiques et administratifs, ou de réseaux spécialisés dans la production clandestine et dans la spéculation à partir de la seconde moitié des années 1970. Par ailleurs, les récurrentes campagnes de renforcement de la lutte contre la criminalité, que ce soit contre le " hooliganisme » en 1966, contre les atteintes à la propriété socialiste en 1968 ou contre le «saccage » (razbazarivanie) de l'économie en 1975 frappaient également la pègre. Enfin, les « parasites " (c'est-à-dire dans la langue soviétique, ceux qui n'exercent pas une activité utile à la société) et les "récidivistes » représentaient avec les «alcooliques» les principales figures de la délinquance invoquées pour expliquer l'augmentation des faits constatés dans une région connue pour le nombre d'établissements pénitentiaires qu'elle abrite. Les rapports évoquaient même parfois l'action de parasites " particulièrement dangereux ${ }^{16}$.

En revanche, l'expression de "criminalité organisée» ou de «mafia» a pu être employée par les dirigeants soviétiques pour désigner les pratiques de détournement de biens publics orchestrées par des segments d'élites politico-administratives régionales. Il a ainsi été question de «mafia du coton» ou de «mafia du poisson ${ }^{17}$. Certains auteurs ont souligné la correspondance entre l'emploi du label de mafia et des pratiques liées à l'expression de revendications d'autonomisation dans la périphérie de $l^{\prime} U n i o n{ }^{18}$. Le terme de «mafia » était cependant également couramment employé pour désigner des secteurs économiques (mafia du commerce ${ }^{19}$ ) ou des branches professionnelles (mafia des taxis).

Trois discours sur la criminalité organisée peuvent donc être distingués : négation de son existence, reconnaissance euphémisée et implicite, instrumentalisation du terme de mafia afin de discréditer un adversaire politique ou un groupe social. L'emploi de ce triple discours était notamment facilité par l'usage de catégories pénales larges et imprécises dans le contexte soviétique. Les campagnes de lutte contre le hooliganisme justifiaient la répression de pratiques illicites extrêmement variées, notamment économiques ${ }^{20}$. L'histoire de l'URSS souligne le recours permanent à des incriminations économiques (sabotage, parasitisme) pour sanctionner des pratiques politiques et réprimer des opposants. Les usages politiques des notions de criminalité organisée et de mafia entraînaient une confusion des représentations qui s'est accentuée à partir de la seconde moitié des années 1980, alors que la glasnost a favorisé l'émergence et la diffusion de débats publics sur les questions criminelles. 


\section{Glasnost : L'ÉMERgENCE d'UNE SITUATION PROBLÉMATIQUE}

La glasnost n'a pas subitement entraîné de débats publics sur la totalité des sujets de société; elle a conduit à une couverture sélective et progressive de situations présentées comme problématiques, dont la criminalité en général et le crime organisé en particulier ont indéniablement fait partie. La transformation des discours sur la criminalité et la constitution d'un problème criminel ont cependant été dues à un faisceau de causes, et non uniquement à l'apparition de débats publics sur ce sujet. La définition pénale de la criminalité a été bouleversée par le changement des règles du jeu économique (mise en œuvre dès 1986 des premières mesures de privatisation de l'activité commerciale, abandon progressif de la planification, ouverture des frontières et libéralisation du commerce extérieur) ${ }^{21}$. Ces réformes ont attiré les différents groupes sociaux qui détenaient les ressources nécessaires pour en profiter (argent, contacts privilégiés avec les administrations) et parmi lesquels se distinguaient notamment les membres du milieu criminel. La société soviétique a alors été confrontée à une période d'incertitude, de coexistence de règles et de valeurs concurrentes, se manifestant notamment par un changement des représentations associées à l'enrichissement personnel, par un approfondissement de la crise économique et par un creusement des inégalités sociales ${ }^{22}$.

Il a fallu attendre le début de 1989 pour que les premières statistiques criminelles soient partiellement publiées dans la presse nationale. Dans un article intitulé «Ces statistiques sont ouvertes pour la première fois", les Izvestia ont fourni des informations homogènes sur douze formes d'infractions, dont l'enregistrement avait significativement augmenté en 1988. L'événement a révélé une situation préoccupante et a causé un « choc » dans l'opinion ${ }^{23}$. C'est cependant un peu plus tôt, à partir de 1988, que se sont multipliés les articles sur la criminalité, en particulier sur le crime organisé, dans la presse et les revues scientifiques. Les rubriques criminelles se sont développées dans les quotidiens russes. Les manifestations les plus violentes de la délinquance ont envahi les programmes télévisés, pénétré dans les foyers et passionné les téléspectateurs. Le rôle et la popularité de l'émission « 600 secondes ", diffusée sur la chaîne de Saint-Petersbourg, ont été considérables ${ }^{24}$. Les scandales locaux, les affaires de corruption, les escroqueries, les meurtres en série et les règlements de compte sanglants transmettaient au téléspectateur l'image indifférenciée d'une société livrée à la violence et au cynisme de dominants impunis. Les règles en vigueur au sein du milieu criminel, abondamment décrites dans les médias, ont pu alors parfois exercer une fascination romantique auprès des jeunes générations. La subite profusion d'informations et d'analyses sur la criminalité a rapidement entrainé la constitution d'un problème mafieux, caractérisé par des interprétations divergentes de sa nature.

\section{Les fondements de la connaissance : définitions et sources}

La criminalité organisée a constitué un problème à partir du moment où sa formulation est devenue l'enjeu de conflits entre différents groupes sociaux. Or, cette préoccupation 
sociale possède des propriétés qui lui donnent des chances de nourrir d'incessants affrontements, sa connaissance s'appuyant sur des sources rares et occultes.

Dans un article consacré à l'Italie, Jean-Louis Briquet a souligné les défis méthodologiques considérables et spécifiques posés par la compréhension de la mafia italienne. D'abord, tout savoir accumulé sur ce sujet représente le produit d'« un travail de dévoilement ${ }^{25}$ : institution occulte, la mafia ne livre pas de sources d'information sur elle-même. En conséquence, la formulation du problème mafieux est marquée par d'incessants conflits entre les acteurs extérieurs impliqués dans sa prise en charge. Enfin, les informations et analyses policières et judiciaires constituent les sources principales pour ceux qui souhaitent analyser le "phénomène mafieux ». Contrairement à la vision dominante, la mafia est « un objet aux contours incertains, une réalité échappant constamment aux définitions qui en sont proposées, un phénomène mouvant et en constante évolution, forçant une évolution correspondante des modèles qui tentent de l'appréhender $»^{26}$. Même si le cas russe s'y soustrait parfois (d'une certaine manière, les «mafieux » eux-mêmes participent à la formulation du problème; la pérennité des organisations criminelles russes demeure incertaine, les plus anciennes n'ayant pas plus de trente ans), ces remarques conservent leur pertinence dans le cas que je souhaite étudier. Elles soulignent en particulier les difficultés inhérentes à la connaissance des pratiques et groupes sociaux que l'expression « crime organisé » désigne.

La constitution du problème du crime organisé à la fin des années 1980 s'est nourrie de la confusion due aux différents usages du terme de mafia dans le contexte soviétique et a contribué à la reproduire, voire à l'étendre. D'une part, même si les termes de mafia et de crime organisé ont beaucoup plus fréquemment été associés au monde de la pègre, ils ont continué à revêtir des significations différentes et à désigner notamment les collusions politico-financières. D'autre part, les frontières de la criminalité organisée ont été obscurcies par l'usage répandu d'autres catégories sans fondements juridiques, telles que criminalité économique ou économie souterraine. L'interpénétration de ces différentes expressions, chacune désignant des ensembles génériques d'infractions, a également contribué à la diffusion de représentations imprécises.

De nombreuses enquêtes sociologiques menées à la fin des années 1980 et au début des années 1990 sur les représentations relatives au crime organisé au sein de la société russe montrent que le terme de mafia est associé au pouvoir, qu'il soit politique ou économique. Le VTsIOM, institut de sondages alors dirigé par le sociologue Youri Levada, après avoir constaté que $51 \%$ des personnes interrogées considèrent que la mafia (ou le crime organisé) est responsable de la majorité de leurs problèmes, pose une seconde question, "Qu'entendez-vous par mafia?», et obtient les réponses suivantes : l'activité de l'économie parallèle (43\%), les bureaucrates dans les ministères et les administrations (36\%), l'appareil du parti-Etat (34\%), les criminels (25\%), les coopérateurs et travailleurs individuels $(18 \%)^{27}$. Le terme de mafia exprime en définitive un sentiment d'injustice sociale, une hostilité à l'égard de l'enrichissement individuel et de la possession de privilèges, une impuissance face aux modes de domination politique et économique. Les termes de profiteur, de spéculateur et de parasite, étroitement liés à la rhétorique léniniste, se confondent avec celui de mafia. Nous verrons plus loin que l'imprécision du sens du terme de mafia alimente les conflits politiques autour de la formulation des enjeux relatifs au crime organisé. 
La multiplication des discours sur la criminalité dans le contexte de la glasnost s'est également traduite par le développement de catégories imprécises, employées dans les publications scientifiques, les journaux ou les forums politiques. Comme la " criminalité organisée ", la "criminalité économique » ou l' "économie souterraine " représentent des ensembles mouvants de pratiques illicites hétérogènes. Ces catégories s'interpénètrent, voire s'englobent les unes les autres, du moins pendant un certain temps. Cette confusion, qui n'est pas spécifique au contexte étudiée ${ }^{28}$, enrichit le répertoire des représentations associées au crime organisé ou à la mafia. Si l'on s'en tient au sondage précédemment présenté, $43 \%$ des personnes interrogées associent la mafia à «l'activité de l'économie parallèle ». Or, cette dernière expression est également fourre-tout.

"Quant à la définition [de l'économie de l'ombre ${ }^{29}$ ], un répertoire extrêmement large et divers d'expressions synonymes peut être mobilisé. On parle d'économie souterraine, seconde, non enregistrée, destructrice, non réglementée, criminelle, petite, fictive, non étatique, marchande et privée, parallèle, noire, informelle, illégale, en dehors de la loi, et la liste n'est pas exhaustive $»^{30}$.

En conséquence, les représentations de la criminalité organisée sont issues de la combinaison de deux variables, relatives aux pratiques et aux auteurs. Les pratiques, plus ou moins diversifiées, peuvent correspondre à des activités économiques clairement illégales ou simplement illégitimes et injustes aux yeux de celui qui emploie le label de « crime organisé »; elles peuvent représenter des sources complémentaires de revenus extrêmement banales dans le contexte soviétique de la fin des années 1980, ou au contraire être liées à des trafics perçus comme criminels (drogues, armes...). Le label de " crime organisé » peut également être attribué à différents groupes sociaux : membres du milieu criminel, entrepreneurs, commerçants, responsables politiques et administratifs...

Durant la perestroïka, le terme de mafia et l'expression de criminalité organisée ont cependant tendu à être prioritairement associés aux activités du milieu criminel, ce qui représente une rupture avec la période précédente. La première interview du lieutenant-colonel de police Alexandre Gourov dans l'hebdomadaire Literatournaïa Gazeta sur la criminalité organisée en Union soviétique a constitué à ce titre un événement de la glasnost et une référence historique ${ }^{31}$. Désormais, la définition de cette forme de délinquance renvoie moins aux élites corrompues qu'au milieu criminel, même si ce dernier est éventuellement connecté aux sphères politiques et administratives. Alexandre Gourov révèle l'existence d'une mafia en Union Soviétique et la présente comme une organisation hiérarchisée, professionnalisée, disposant de contacts solides avec des agents de l'Etat. Cette interview suscite de nombreuses réactions ${ }^{32}$ et accélère le processus de création, au sein du ministère de l'Intérieur, d'une direction de la lutte contre la criminalité organisée, à la tête de laquelle il est luimême nommés ${ }^{33}$. Les réactions à l'article d'Alexandre Gourov (des témoignages et des analyses sur la place qu'occupe le crime organisé dans la société soviétique) frappent par la diversité des pratiques qu'elles décrivent: il est question de règlements de comptes avec des armes à feu, de rackets de coopératives, de jeux de carte et de la hiérarchie criminelle traditionnelle. Un point de vue officiel sur le sujet, présenté par le chef de la direction générale des recherches criminelles du ministère de l'Intérieur, indique que :

« de 1986 à 1988, des centaines de groupes particulièrement dangereux, agissant dans le domaine économique, commettant des vols, vols à découvert, vols avec 
violence et rackets ont été mis hors d'état de nuire par les services du ministère de

l'Intérieur $»^{34}$.

Ce discours illustre les modes de fonctionnement de la bureaucratie policière. La création d'une nouvelle et imprécise catégorie d'infractions s'accompagne presque toujours de résultats immédiats, constitués dans la pratique par agrégation de pratiques diverses. Les "groupes particulièrement dangereux ", loin d'être des syndicats du crime, peuvent tout à fait représenter le produit d'ententes entre deux ou trois personnes, motivées par l'appât du gain, sans aucun contact avec la pègre.

L'interview d'Alexandre Gourov dans la Literatournaïa Gazeta inaugure également les principaux canaux d'expertise sur le crime organisé. L'information sur cette forme de délinquance est collectée et diffusée par les services opérationnels et scientifiques des institutions policières et judiciaires. Jusqu'au milieu des années 1990, presque tous les discours présentés comme scientifiques sur le crime organisé proviennent des instituts de recherche du ministère de l'Intérieur, de la Prokouratoura ${ }^{35}$ générale de la Fédération de Russie ou d'autres institutions répressives. Ce monopole, qui s'explique notamment par le mode de constitution historique de la criminologie soviétique ${ }^{36}$, touche d'autres champs disciplinaires, juridique ${ }^{37}$, économique, voire sociologique. Durant les années 1990, les ouvrages et les articles de revues sociologiques sur le crime organisé sont principalement écrits par des chercheurs rattachés à l'Intérieur ou à la Prokouratoura $^{38}$.

Les problèmes posés par ce monopole sont principalement méthodologiques, car les sources sur l'évolution du crime organisé, produites au sein des services policiers et judiciaires, sont rarement dévoilées. L'opacité des sources d'information est encore renforcée par les modes de production des rapports de police sur la criminalité. Tenus de répondre aux attentes de leur hiérarchie, souvent exprimées de manière quantitative, les services locaux de police rédigent des rapports d'activité conformes aux injonctions reçues. Selon les priorités, ils affichent des indicateurs d'activité (nombre de faits constatés, taux d'élucidation) plus ou moins proches du travail effectué, en jouant sur les qualifications, en extorquant les aveux ou en fabriquant les affaires relevant des catégories d'infractions privilégiées par la hiérarchie ${ }^{39}$. Dans ces conditions, l'apparent respect des consignes reçues afin de « renforcer la lutte contre le crime organisé » peut dissimuler des pratiques qui contredisent les orientations générales de la politique menée.

En Russie comme ailleurs, les sources policières sur le crime organisé ont traditionnellement tendance à surestimer le caractère organisé des groupes qui se consacrent à une ou plusieurs activités criminelles ${ }^{40}$. Cependant, dans le contexte considéré, le poids des sources policières n'est pas contrebalancé par des travaux ethnographiques ${ }^{41}$, par une analyse rigoureuse de dossiers judiciaires relatifs au crime organisé ou par des récits de repentis. Les chercheurs qui ne sont pas rattachés aux institutions répressives n'ont guère accès aux sources policières. En conséquence, les seules sources alternatives disponibles sont des investigations journalistiques. Ces enquêtes se multiplient dès la perestroïka et connaissent un grand succès, notamment lorsqu'elles décrivent la structuration et l'activité des organisations criminelles dans les grandes villes de Russie ${ }^{42}$. De qualité inégale, elles ne dévoilent guère leurs sources d'informations et s'effectuent selon les logiques propres aux investigations journalistiques. Compte tenu de l'impact de la privatisation et de la restructuration des médias en Russie, il demeure difficile de comprendre les raisons qui poussent un 
quotidien national à dévoiler une affaire retentissante en rapport avec le crime organisé. L'accumulation au cours des années 1990 des kompromaty (documents compromettants) qui ternissent la réputation d'un personnage public, laisse ouvertes les interrogations sur les conditions de mise en visibilité et de publicité des affaires qui défraient la chronique.

Les deux sources fondamentales de la connaissance du crime organisé, entendu comme milieu criminel ou pègre, sont donc policières et journalistiques. Elles sont en interaction pour deux raisons. D'une part, elles s'étayent l'une l'autre. Les travaux des instituts de recherche scientifique du ministère de l'Intérieur ou de la Prokouratoura générale de la Fédération de Russie sont souvent construits à partir de sources confidentielles et de références bibliographiques à des articles de presse. D'autre part, elles partagent souvent une approche similaire du crime organisé, approche fondée, à tort ou à raison, sur l'indignation et le catastrophisme. Or, ces sources alimentent tous les discours sur le sujet - politiques, journalistiques ou scientifiques - sans pour autant qu'ils soient toujours identiques. A partir du répertoire étendu des représentations associées à la mafia ou au crime organisé, émergent progressivement deux formulations concurrentes du problème, qui donnent lieu à des affrontements dans les arènes politiques, économiques et scientifiques.

\section{La polarisation du problème criminel}

«L'explication d'un problème social chronique ne peut jamais être unanimement approuvée. [...]Elle vise à intensifier la polarisation, en maintenant les divisions entre les camps $»^{43}$. Les deux interprétations du problème criminel, bien qu'inégalement diffusées au sein de la société, reflètent et consolident l'opposition politique majeure qui caractérise le jeu politique russe à la fin des années 1980 et au début des années 1990, entre " conservateurs » et " réformateurs ». Cette opposition est marquée par une divergence d'appréciation quant à la formulation du problème mafieux, à l'analyse de ses causes et aux réponses à fournir. L'interprétation du problème criminel cristallise les divisions de la scène politique, car la question du crime organisé est notamment liée aux débats plus généraux sur la réforme économique à entreprendre dans le pays. A ce titre, l'appréciation des effets des premières mesures de privatisation de l'activité commerciale, notamment de la loi sur les coopératives de 1988, a joué un rôle décisif, en liant les réformes entreprises à des enjeux criminels ${ }^{44}$. Le problème du crime organisé est également, quoique plus indirectement, construit en relation avec les débats sur la transition politique et la démocratisation.

Chacune des interprétations réunit des acteurs politiques, économiques, des journalistes et des chercheurs, qui produisent des discours se validant mutuellement et aboutissant à la constitution d'un corps de connaissance sur ce sujet. Chaque corps de connaissances est fondé sur des considérations idéologiques crédibilisées par des experts mobilisant des discours scientifiques. Les acteurs répressifs et les scientifiques issus des institutions répressives ne se partagent pas entre les deux camps et promeuvent presque toujours une vision alarmiste du crime organisé.

Pour les uns, le crime organisé est d'abord perçu comme une conséquence des réformes économiques lancées en U.R.S.S. depuis la seconde moitié des années 1980. Il est étroitement lié aux opportunités d'enrichissement personnel offertes par la privatisation du secteur commercial. Ceux qui profitent des réformes économiques 
pour s'enrichir sont des éléments criminels dominés par l'égoïsme et le cynisme. Cette approche centrée sur l'opportunisme des criminels cède rapidement la place à la conviction selon laquelle la mafia participe, grâce à son insertion dans les milieux dirigeants politiques et économiques, à la création même des nouvelles règles du jeu économique. Les réformes économiques représenteraient alors la traduction en programme politique des intérêts mafieux. Le crime organisé menace la société soviétique dans son ensemble, en multipliant les victimes et en détournant les jeunes de valeurs vertueuses.

Selon cette représentation, l'augmentation de la criminalité caractérise la perestroïka. Produite par les réformes gorbatcheviennes, elle représente un phénomène central dans la société russe. Le crime organisé, la criminalité économique et l'économie souterraine sont des expressions différentes qui désignent un même phénomène: l'activité des organisations criminelles, considérées comme un acteur social unique poursuivant un objectif commun à toutes ses composantes. Le crime organisé vise à déstabiliser l'économie et l'Etat et à acquérir du pouvoir au sein des instances nationales de décision. Il est alors question d'économie criminelle ${ }^{45}$, d'Etat mafieux, de "grande révolution criminelle ${ }^{46}$. Cette perception débouche éventuellement sur la dénonciation d'un complot, susceptible de convaincre une grande partie de la population. L'expansion internationale d'une organisation criminelle est nécessairement inscrite dans son évolution ${ }^{47}$. Seule une répression déterminée et indifférenciée est susceptible de contenir cette vague criminelle.

« Non, il n'y a pas d'un côté des crimes graves et de l'autre des infractions anodines. Toute infraction représente le même danger pour la société. Il n'y a pas de petits et de grands voleurs, de menu fretin et de gros poissons. Un voleur est un voleur. Et il doit croupir en prison $»^{48}$.

Or, dans la mesure où les milieux politiques et économiques, soi-disant soucieux de démocratie et d'économie de marché, sont infiltrés par des éléments criminels, des mesures autoritaires doivent être prises.

«L'adoption de mesures décisives exigera sans doute une ferme opposition à l'anarchie et au chaos que certains, dans leur propre intérêt, et non dans celui de l'Etat, tentent de présenter comme une manifestation de la démocratie. Une authentique démocratie n'a rien de commun avec une telle approche. La démocratie doit être le garant de la renaissance juridique de l'Etat, qui seule permet de défendre les droits des individus, de la société et de la nation. L'action du Président et du Soviet suprême, précisément orientée vers la défense de ces droits, consiste à confier aux organes du pouvoir d'Etat la tâche d'attaquer frontalement la criminalité et la corruption, menace fondamentale pour les réformes en Russie. La Commission interdépartementale du Conseil de sécurité dispose des compétences nécessaires pour que le peuple ressente que le temps des slogans et des annonces est révolu et qu'une offensive implacable sera menée contre la criminalité ${ }^{49}$.

Dans la mesure où le terme de « démocratie » est subverti par les réformateurs, agents des intérêts mafieux et /ou étrangers ${ }^{50}$, seules des mesures dictatoriales sont susceptibles de sauver le pays.

«Aujourd'hui, selon moi, seule la terreur peut stopper la vague criminelle [...]. Seule une main de fer peut stopper la criminalité, rendue à un niveau critique [...]. Aujourd'hui, nous sommes contraints de prendre les mesures les plus extrêmes, les plus astreignantes pour tenter de stopper la vague de la criminalité $»^{51}$.

Une même interprétation du crime organisé réunit donc les services répressifs, la plupart des experts scientifiques, les hommes politiques conservateurs, certains journalistes, certains personnages publics et les chercheurs opposés aux réformes néo- 
libérales. Cette vision est sans doute partagée par une grande partie de la population, si l'on en croit les sondages déjà mentionnés. L'homogénéité des discours est renforcée par des procédés de validation mutuelle. Les chercheurs et experts justifient leurs thèses en s'appuyant sur des déclarations politiques et des articles de presse, et inversement. Stanislas Gororoukhine est cité dans des manuels de droit ${ }^{52}$ ou dans des ouvrages à prétention scientifique sur le crime organisé5 ${ }^{53}$; il multiplie, quant à lui, les éloges d'Alexandre Routskoii ${ }^{54}$, etc.

Les intérêts des différents acteurs participant à la constitution d'un corps de connaissances homogène sur la criminalité organisée ne sont cependant pas forcément similaires. Pour les agents répressifs, il s'agit d'abord d'obtenir des moyens supplémentaires. Au sein du ministère de l'Intérieur, les services de lutte contre la criminalité organisée acquièrent une importance croissante, au point de devenir une direction générale, isolée par rapport à tous les autres services de police judiciaire, disposant d'une autonomie financière accrue. L'organisation territoriale de cette direction est spécifique : le champ d'activité de chaque direction régionale s'étend à plusieurs sujets de la Fédération de Russie afin d'éviter les collusions entre élites locales et services opérationnels ${ }^{55}$. Les services de lutte contre la criminalité organisée acquièrent progressivement, au cours des années 1990, des compétences qui non seulement empiètent sur celles des services traditionnels de police judiciaire, mais qui exposent ces derniers, suspectés d'entretenir des connivences avec les pouvoirs locaux, à leur surveillance. Par ailleurs, la lutte contre le crime organisé fait désormais partie des attributions des services de sécurité ${ }^{56}$. Pour les hommes politiques, il s'agit de répondre à l'indignation et aux craintes d'une grande partie de la population, en affichant une implacable détermination et en proposant soit un retour à l'ordre ancien, soit un programme de transition plus graduel.

Ce discours se maintient au cours des années 1990. Même s'il est de moins en moins question de promouvoir la restauration du régime communiste, de nombreux dirigeants politiques continuent de construire leur légitimité sur la détermination dont ils feraient preuve s'ils étaient au gouvernement. La dénonciation des collusions entre intérêts politiques, économiques et mafieux demeure extrêmement présente dans le jeu politique russe ${ }^{57}$. La mafia continue d'être présentée comme le principal détenteur du pouvoir dans le pays ${ }^{58}$.

Face à cette interprétation de la criminalité organisée, se construit progressivement une formulation alternative du problème. Principalement relayée par des hommes politiques impliqués dans la conception des réformes économiques de 1992, certains entrepreneurs, journalistes et chercheurs, elle propose une analyse différente des causes et de l'ampleur du problème criminel. Les relations entre criminalité et économie doivent d'abord être cherchées dans le passé soviétique, alors que les pénuries et la planification favorisaient l'exploitation des difficultés matérielles. Les pratiques illicites constatées lors du processus de privatisation sont expliquées de deux manières. D'une part, elles sont dues au caractère incomplet des réformes menées en 1992. La plupart des entrepreneurs qui sont considérés comme des "mafieux " ne demandent qu'à travailler dans l'honnêteté: confrontés à l'absurdité des semiréformes, d'une politique fiscale inadaptée et des conflits entre différents niveaux de régulation, ces innovateurs sont contraints de recourir à des pratiques illicites. D'autre part, elles sont de toute façon moins généralisées que si la privatisation «spontanée " ${ }^{59}$ s'était poursuivie ou si rien n'avait été fait pour changer la nature des droits de 
propriété. Pour A. Tchoubaïs, leader politique et artisan des privatisations, «le niveau de criminalité que nous connaissons aujourd'hui est dérisoire par rapport à celui qui aurait été atteint si les privatisations n'avaient pas été menées $»^{60}$. Selon cette approche, les pratiques économiques criminelles ne représentent qu'un épiphénomène au regard des efforts effectués pour changer complètement les règles du jeu économique. Emblématiques d'une période d'accumulation primitive du capital, elles sont vouées à disparaître progressivement.

En conséquence, les expressions telles que "criminalité organisée ", "criminalité économique » ou « économie souterraine » doivent être employées avec prudence car elles rassemblent des pratiques diverses, plus ou moins justifiées. Les réformateurs développent notamment un discours sur l'économie souterraine à partir de la fin des années 1980 qu'il importe de présenter, car il va alimenter les représentations sur l'étroitesse des liens qui les attachent au milieu criminel. Comme il a déjà été observé, le développement du secteur coopératif à partir de 1987, première forme importante de privatisation de l'activité commerciale, a souvent été perçu comme une mesure bénéficiant au milieu criminel, voire initiée par ce dernier. En 1990, les dirigeants soviétiques, à la recherche de moyens de réformer l'économie, commandent à l'académicien S. Chataline un rapport intitulé "La transition vers le marché. Conception et programme ». Ce texte majeur ${ }^{61}$, appelé "Programme des 500 Jours ", consacre un chapitre entier à la question de l'économie souterraine. Il est d'abord affirmé que cette économie disparaîtra lorsque le marché remplacera le dirigisme étatique :

«nombreux sont ceux qui lient l'essor de l'activité économique souterraine et illégale à la transition vers le marché. Or, c'est justement le contraire qui va se produire. L'économie souterraine est le complément indispensable de l'économie soviétique. Plus les mécanismes de distribution des marchandises se substituent à leur commerce normal, plus le champ d'activité de l'économie souterraine s'élargit. La transition vers le marché constitue le moyen le plus efficace pour la liquider ${ }^{62}$.

Mais le programme contient une idée plus surprenante, selon laquelle il faut chercher à attirer et exploiter les capitaux souterrains :

« l'ampleur de l'économie souterraine[...] revêt une importance particulière car le programme de transition vers le marché prévoit logiquement d'utiliser les capitaux souterrains afin de servir les intérêts de tous. Ils représentent en effet une ressource de premier plan pour garantir le succès de la réforme $"^{63}$.

D'après les auteurs, l'introduction de mécanismes de marché devrait favoriser l'intégration de $90 \%$ des capitaux « souterrains » dans l'économie officielle ${ }^{64}$. Cette idée réapparaît tout au long des années 1990. Elle offre d'abord un argument pour justifier les privatisations ${ }^{65}$. Elle répond ensuite au besoin d'investissements pour sortir de la crise économique. Au milieu des années 1990, des clubs réformateurs proposent de légaliser les revenus issus de l'économie souterraine afin de lutter contre le blanchiment et la fuite des capitaux ${ }^{66}$. Ces discours s'accompagnent généralement d'une distinction entre acteurs vertueux de l'économie souterraine (des entrepreneurs que les contraintes juridiques et administratives empêchent de mener une activité complètement légale) et acteurs criminels. Il conviendrait de « distinguer nettement les capitaux du milieu criminel et des entrepreneurs, puis d'intégrer cette distinction dans la législation pénale sur la lutte contre le crime organisé et la corruption ${ }^{67}$.

D'où l'idée, parfois exprimée, d'un moratoire sur l'économie souterraine. Cette idée est fondée sur le constat selon lequel, d'une part, il est impossible de distinguer des 
capitaux selon l'identité de leur détenteur et, d'autre part, l'intérêt général doit conduire à dépasser tout jugement moral sur leur origine. Une fois le moratoire décrété, de nouvelles règles du jeu économique seraient mises en place. Cette idée est relayée par des personnalités telles qu'Edouard Rossel, le gouverneur de la région de Sverdlovsk. L'amnistie conduirait selon lui à un redressement du secteur industriel et à une amélioration des conditions de vie de la population ${ }^{68}$. Selon le même raisonnement, les ententes avec le milieu criminel ne doivent pas être condamnées a priori : "si j'apprenais qu'untel est un mafieux, je l'inviterais à discuter et lui proposerais de travailler dans la légalité ». Le terme de « mafia » doit être relativisé car il pourrait tout aussi bien être employé à propos des services de sécurité ou des dirigeants du pays ${ }^{69}$. Ce discours relativiste et utilitariste se retrouve également chez Vladimir Jirinovski qui inclut dans sa liste lors des élections législatives de décembre 1999 des figures notoires du milieu criminel, considérant que les richesses et le pouvoir détenus par ces caïds sont susceptibles d'aider au développement du pays ${ }^{70}$.

Tous ces discours suscitent de vives réactions. Le Programme des 500 Jours est notamment considéré comme un événement fondateur, révélateur de la permissivité dont ont pu faire preuve les instigateurs des réformes économiques, voire de leur complaisance à l'égard du milieu criminel.

«Dans le Programme des 500 jours, le slogan sur la conversion et la légalisation des capitaux souterrains comme moyen d'assurer le financement des réformes a joué un rôle extrêmement négatif dans la lutte contre les mafias. Son impact sur l'action menée par les services répressifs pour encadrer la mise en œuvre des lois sur l'entreprise, sur la commercialisation, sur la fiscalité et sur l'interdiction des monopoles a été particulièrement fort. Au total, le transfert de la propriété a revêtu dans les années 1990 un caractère non civilisé, criminel $»^{71}$.

Cette perception du programme des 500 jours doit être comprise comme l'un des effets de la polarisation du problème criminel en Russie. Les champions de la détermination face au crime organisé interprètent toute tentative de différencier les pratiques, de comprendre cette expression imprécise ou de relativiser son impact comme une preuve manifeste du pouvoir des mafias. Cette thèse est d'ailleurs alimentée par les discours que tiennent des membres d'organisations criminelles sur leur propre activité, dans les entretiens anonymes qu'ils accordent à la presse au début des années 1990. Selon les textes de présentation de ces entretiens, l'initiative vient en général des représentants du milieu criminel, exaspérés par l'incompréhension dont leur activité fait l'objet et par la multiplication des discours à leurs yeux illégitimes sur la mafia et l'économie de l'ombre :

«Ce n'est pas moi qui ai besoin de cette interview, c'est vous, vous tous, citoyens serviles de l'Empire. Les officiers du ministère de l'Intérieur et les professeurs d'université vous trompent, en débitant chaque jour à la télévision et dans les journaux des sornettes sur notre compte. [...] Si nos respectables fonctionnaires se permettent, avec des grands airs d'experts, de disserter sur ce qu'ils appellent mafia et économie de l'ombre, alors pourquoi ne prendrions-nous pas la parole sur ces sujets? Tout ce qui est dit, toutes ces études prétendument scientifiques qui entraînent une grande confusion dans le cerveau des citoyens, toute cette propagande sur la lutte contre le crime organisé - tout cela n'est que sottises, tromperie et simulation $»^{72}$.

Fictifs ou non, ces entretiens, souvent publiés dans des journaux ou revues proches des milieux réformateurs, tendent généralement à relativiser ou à récuser le terme de mafia. Ceux qui enfreignent la loi distinguent leur activité de celles qu'ils jugent 
moralement condamnables. Selon ce raisonnement, mafia et crime organisé doivent être d'abord associés aux détenteurs du pouvoir politique.

«Pour parler franchement, j'en ai marre de ce qui est dit et écrit sur nous. On nous colle la responsabilité des pires crimes commis sur terre [...]. D'accord, nous enfreignons la loi, mais jamais il ne nous viendrait à l'idée de voler un ingénieur peu fortuné ou un vieillard misérable. Ces crimes odieux, c'est notre premier ministre qui les commet [...]. Nous, on se contente de partager ce qui a déjà été volé par des voyous $»^{73}$.

De même, ceux qui œuvrent pour une humanisation de la justice pénale ou une amélioration des conditions de vie des détenus se heurtent parfois à un mur d'incompréhension. C'est notamment le cas d'une association fondée par d'anciens dissidents au moment de la perestroïka et destinée à soutenir les détenus condamnés pour des pratiques considérées selon la législation pénale soviétique comme des "crimes économiques» alors qu'elles auraient été encouragées dans d'autres contextes. Lorsqu'au cours d'une réunion publique en 1990 sur les libertés économiques et les droits de l'homme, cette association expose les dangers d'une réforme économique dissociée d'un changement profond de la législation pénale et propose de nouvelles incriminations, l'un des participants observe que «selon toute évidence, dès que cette idée paraîtra dans la presse, on nous traitera de complices de la mafia et de pourris ${ }^{74}$. Les plaidoyers pour une réhabilitation des "gestionnaires soviétiques condamnés » illustrent pour ceux qui promeuvent une lutte sans merci contre le crime organisé les connivences à l'œuvre entre élites politiques, économiques et milieu criminel. Les mafieux sont si puissants qu'ils téléguident des lobbies fondés pour promouvoir l'amnistie et la réhabilitation de leurs collègues incarcérés... ${ }^{75}$

Ces exemples montrent à quel point les positions relatives au problème criminel s'exaspèrent. Tel qu'il est construit, ce problème entraîne des formulations concurrentes étroitement liées à des représentations idéologiques plus générales sur le changement politique et économique souhaitable pour la Russie. Dès lors, le thème du crime organisé ne représente qu'un élément du conflit qui oppose deux visions du monde radicalement différentes. La fermeté est interprétée comme une atteinte aux libertés, un penchant à l'autoritarisme, une nostalgie du totalitarisme, alors que la compréhension et le relativisme sont présentés comme la manifestation d'une irresponsabilité, voire d'une connivence avec les intérêts mafieux. Les débats sur le crime organisé sont davantage motivés par des préoccupations politiques que par le souci d'améliorer l'efficacité des politiques pénales. L'imprécision de la définition du crime organisé, loin de contraindre la décision politique, représente, au moins pendant un certain temps, une ressource susceptible d'aider à la construction d'une légitimité, à la déstabilisation d'un adversaire ou à la dénonciation d'un groupe social. Les discours scientifiques sur cette forme de délinquance relaient ces antagonismes et ne permettent pas au débat de s'élever, par exemple en critiquant les sources disponibles, en proposant des méthodes de recherche susceptibles de s'y substituer ou en dénonçant les divers usages politiques du problème criminel.

Ce constat est certes historiquement ancré dans un contexte de changement profond de la société russe ; comme nous le verrons, les discours scientifiques sur le crime organisé tendent à se différencier à partir de la seconde moitié des années 1990. Il reste que les perceptions décrites, opposant un discours dominant sur la gravité du problème et la nécessité d'un retour à l'ordre à des positions relativistes souvent inscrites dans une rhétorique néo-libérale, orientent le débat occidental sur la mafia russe. 


\section{« Les Russes arrivent ! ${ }^{76}$. Regards occidentaux sur la mafia russe}

Pour comprendre l'importance accordée dans le monde occidental au crime organisé russe au début des années 1990, il faut d'abord mentionner quelques éléments propres au contexte américain. L'échec rencontré par les différentes stratégies de lutte antidrogues menées au cours des années 1980 a progressivement conduit le gouvernement américain à considérer que seule une action internationale était en mesure de répondre à cet enjeu. La lutte anti-blanchiment apparaît à la fin des années 1980 (Convention de Vienne 1988, Sommet de l'Arche et création du Gafi en 1989) comme une réponse nouvelle et appropriée, réorientant la lutte anti-drogues vers une action menée contre les capitaux issus des trafics ${ }^{77}$.

La chute du mur de Berlin s'effectue donc dans un contexte où les flux criminels transnationaux sont déjà perçus comme des menaces pour la sécurité intérieure américaine. L'illusion euphorique d'une transition rapide et réussie de la Russie et des autres républiques soviétiques vers l'économie de marché et la démocratie cède rapidement la place à une inquiétude issue d'un double constat : la désagrégation d'une formidable puissance militaire et le pouvoir croissant du crime organisé au sein de la société post-soviétique. Ces peurs sont alimentées par les informations en provenance de Russie. Le crime organisé contrôle l'économie russe et acquiert des positions importantes au sein des institutions politiques et des administrations, comme le répètent à l'envi les dirigeants politiques, les services répressifs, les journalistes et les scientifiques, bien informés sur les pratiques en vigueur dans leur pays. Les nombreux travaux universitaires et journalistiques américains qui s'appuient sur les sources policières et les médias russes relaient cette opinion. Les ouvrages sur les « camarades criminels $^{78}$ » ou sur $~ « l ' e m p i r e$ de toutes les mafias $»^{79}$ connaissent un certain retentissement dans le monde occidental.

L'idée selon laquelle l'expansion mondiale de la mafia russe est inscrite dans son développement naturel s'impose rapidement. Cette conviction est d'abord fondée sur des représentations relatives aux organisations criminelles en général : la volonté de profit conduit ces entreprises à la recherche de monopoles, dans des secteurs d'activité ou sur des territoires donnés. La mafia russe semble ensuite particulièrement performante, combinant les compétences de criminels endurcis, d'entrepreneurs et de juristes qualifiés attirés par les perspectives de profit, et d'anciens militaires ou d'agents du KGB parfois mus par un irrépressible besoin de revanche et disposant de technologies sophistiquées puisées dans les décombres de la superpuissance. Ses activités sont non seulement celles de toute organisation criminelle (trafics de drogues, d'armes, d'êtres humains, blanchiment), mais aussi liées à la spécificité du contexte post-soviétique: le trafic de matières nucléaires ou d'armes secrètes donne une nouvelle ampleur au danger que représente la mafia russe ${ }^{80}$.

Pour de nombreux experts, l'apparition de la mafia russe dans le jeu mondial parachève la formation du «crime organisé mondial» ou "transnational» (global organized crime,transnational organized crimeouT.O.C.), réseau de mafias liées par des intérêts communs. Deux catégories d'experts alimentent notamment cette représentation: agents des services de renseignement, de contre-espionnage et de lutte contre la grande criminalité; vedettes du journalisme d'investigation. Parmi ces dernières, la 
plus célèbre est vraisemblablement Claire Sterling, dont les ouvrages constituent une référence incontournable sur le sujet. Auteur à succès d'investigations journalistiques, elle avait publié dans les années 1980 un ouvrage sur les réseaux terroristes mondiaux ${ }^{81}$. Au début des années 1990, elle décrit les sommets tenus par une sorte de G6 mafieux, composé d'Américains, de Colombiens, d'Italiens, de Japonais, de Chinois de Hong Kong et de Russes, durant lesquels il est question de partage du monde et de création d'un "Worldwide Mafia International, the first in history", d'un "CrimeIntern " se substituant au Komintern ${ }^{82}$. La mafia mondiale est présentée comme un acteur unique, un ennemi à la mesure du monde occidental, comparable à l'ancien bloc communiste ${ }^{83}$.

Au milieu des années 1990, cette interprétation est également relayée par certains milieux politiques, pour des raisons différentes. Les acteurs qui participent à une formulation commune d'un problème social ne poursuivent en effet pas nécessairement les mêmes intérêts ; la constitution de coalitions d'intérêt obéit à des raisons conjoncturelles. Dès le milieu des années 1990, des élus républicains et des think-tanks proches de ce parti se saisissent du dossier sur le crime organisé russe pour dénoncer la politique gouvernementale menée à l'égard de la Russie. Le rapport «Russian organized crime» de 1997 du Center for Strategic International Studies, think-tank regroupant des politiciens, des militaires, des agents des services de renseignement et des "experts » civils (dont Claire Sterling) ${ }^{84}$, est particulièrement emblématique de ce raisonnement. La Russie est en passe de devenir un Etat mafieux, un «rogue state» selon la terminologie aujourd'hui en vigueur. Il convient de «réorienter la politique diplomatique et commerciale américaine» car soutenir l'économie de ce pays revient à subventionner les criminels qui par ailleurs cherchent à consolider leur emprise sur les Etats-Unis ${ }^{85}$.

Tous ces discours s'appuient sur des sources russes. Les informations publiées par les quotidiens russes communistes alimentent paradoxalement les opinions exprimées par des personnalités politiques républicaines sur le crime organisé. De même, les sources policières russes sont utilisées sans précaution par ceux qui reconnaissent par ailleurs volontiers les difficultés auxquelles est confronté le ministère de l'Intérieur en Russie (corruption, défaillance des modes de construction des indicateurs d'activité). Cette circulation, parfois imparfaite, est bien illustrée par les chiffres donnés sur la place occupée par le crime organisé dans l'économie russe :

«Le ministère de l'Intérieur russe estime que $40 \%$ des entreprises privées, $60 \%$ des entreprises d'Etat et $50 \%$ à $85 \%$ des banques sont contrôlées par le crime organisé $»^{86}$.

«Dans l'industrie, le commerce, la banque et les services, le crime organisé possède ou contrôle directement environ $85 \%$ des entreprises privées $»^{87}$.

« De $70 \%$ à $80 \%$ des entreprises privées ou privatisées et des banques commerciales sont contraintes de payer groupes criminels, agents publics corrompus et racketteurs $»^{88}$.

«En 1994, un rapport rédigé par le Centre d'analyse économique et sociale de la présidence de Russie estimait que presque toutes les petites entreprises, et 70 à $80 \%$ des grandes sociétés et des banques, payaient un tribut à une mafia [...]. La situation ne semble guère s'être améliorée à l'heure où j'écris ces lignes (1997). Selon les Izvestia, 41000 entreprises industrielles, $50 \%$ des banques et $80 \%$ des sociétés en partenariat avec des investisseurs étrangers ont un lien avec le crime organisé ${ }^{89}$.

Les nuances distinguant chaque citation pourraient entraîner un long développement. Il importe davantage de constater que ces chiffres, qui ne signifient rien en l'absence de 
précisions sur la nature du « contrôle » opéré par le « crime organisé », ont valeur de preuve dans de longs développements consacrés à la puissance de la mafia russe.

De nombreux travaux scientifiques, écrits par des sociologues ou des criminologues, s'indignent de la progression du crime organisé mondial, notamment de sa composante russe, et souhaitent sensibiliser les opinions publiques aux dangers qu'il représente pour les démocraties occidentales. Ils contribuent ainsi à un processus de validation mutuelle des discours politiques, journalistiques et scientifiques, de constitution d'une unanimité objective sur la gravité de l'expansion mondiale du phénomène mafieux. Ils considèrent en effet que la mafia mondiale est composée de différentes organisations criminelles fondées sur une appartenance nationale (mafia italienne, cartels colombiens, yakusa japonaise, triades chinoises, mafia russe...) et unies par des intérêts communs. Ils s'appuient par ailleurs sur les sources habituelles, suffisamment nombreuses et alarmantes pour que leur fiabilité ne soit pas mise en doute. Ainsi, Manuel Castells, dans le troisième volume de la trilogie "L'ère de l'information ", consacre un chapitre à la «filière perverse : l'économie criminelle mondialisée ». Il présente notamment les analyses de Claire Sterling:

«On trouvera un excellent panorama de l'expansion mondiale du crime organisé dans Sterling (1994): bien qu'on[lui]ait reproché de verser un peu trop dans le sensationnalisme, aucun des faits qu'elle rapporte, au terme de patientes enquêtes, n'a été, que je sache, contesté $»^{90}$.

Dans un passage assez déconcertant, le sociologue cherche à justifier son intérêt pour le global organized crime :

«Tout le monde s'accorde à reconnaître l'importance du phénomène, sur lequel on dispose d'une assez abondante documentation, réunie par la presse et par certains organismes internationaux. Toutefois, sociologues et économistes hésitent encore à l'étudier, au motif que les données manquent de fiabilité et que la recherche du sensationnel biaise trop souvent l'analyse. Je vois les choses autrement: si un phénomène est reconnu pour être une dimension fondamentale de nos sociétés et du nouveau système mondialisé, il convient d'utiliser toute la documentation disponible pour étudier la façon dont ces activités criminelles s'insèrent dans la vie économique et sociale à l'échelle du globe $»^{91}$.

Le volume des documents disponibles sur un sujet donné justifie donc sa pertinence. L'accumulation de sources se validant mutuellement conduit à donner une réalité indiscutable au phénomène mafieux. En reprenant les chiffres et sources en circulation sans les soumettre à une analyse critique, le sociologue contribue à leur conférer une nouvelle validité.

L'ouvrage de Jean Ziegler, sociologue et parlementaire, sur « les seigneurs du crime. Les nouvelles mafias contre la démocratie » illustre de manière tout aussi pertinente le processus de circulation des discours sur le crime organisé. En fondant son travail sur des données policières, criminologiques et journalistiques, ainsi que sur les rapports d'activité des commissions anti-mafia au sein des institutions politiques de nombreux pays européens, le sociologue se heurte à l'impossibilité d'apporter ses propres sources à la connaissance du phénomène mafieux.

«Mes collaborateurs et moi-même ne sommes que de modestes sociologues, au courage limité, et non de grands et téméraires journalistes d'investigation. Tenter d'interviewer les Buyuk-baba turcs, les seigneurs pathans du Khyber, les Vor v zakone russes? Rencontrer leurs soldats, diffuser au sein de leurs organisations nos questionnaires ou, pis, tenter une observation participante en nous fondant dans le milieu? Exclu! Nous ne pouvions mener les classiques enquêtes sociologiques sur le terrain. Nous voulions rester en vie $»^{92}$. 
Deux remarques peuvent être opposées à cette approche. D'une part, comme nous le verrons dans les contributions de sociologues russes à ce numéro, les enquêtes de terrain sur le crime organisé ne sont pas réservées aux journalistes d'investigation. D'autre part, s'il est compréhensible de ne pas avoir envie d'approcher du milieu criminel, pourquoi relayer les discours policiers ou politiques sans analyser leurs conditions de production?

Les récents ouvrages ou manuels sur le crime organisé s'inscrivent dans la continuité de ce qui s'écrit sur ce sujet depuis le début des années 1990. Dans l'un d'entre eux par exemple, le chapitre sur l'espace post-communiste aborde notamment la question du trafic de matières nucléaires. Même si l'auteur évoque la rareté des sources disponibles, « les infractions nucléaires [" nuclear offenses »] représentent un nouveau phénomène associé au crime organisé. Le danger que des groupes terroristes ou des individus criminels utilisent ou menacent d'utiliser des matières nucléaires pour commettre des crimes graves est réel $»^{93}$. L'amalgame entre criminalité organisée et terrorisme nécessiterait un développement spécifique: fréquemment exploité dans le monde occidental ${ }^{94}$, il est alimenté au cours des années 1990 par les deux guerres de Tchétchénie, présentées à la fois comme des ripostes anti-terroristes et des opérations de lutte contre la mafia tchétchène ${ }^{95}$. Il n'est pas surprenant de retrouver dans les pages consacrées à la Russie les chiffres des Izvestia selon lesquels « la mafia russe contrôle aujourd'hui 70 à $80 \%$ de la totalité du commerce privé et du secteur bancaire $\aleph^{96}$. Il est en revanche plus original d'apprendre que $31 \%$ des membres des 3500-4000 groupes criminels en vigueur en Russie sont titulaires d'un diplôme universitaire ${ }^{97}$.

Face à ce discours dominant, des interprétations alternatives du problème criminel en Russie peuvent être trouvées. Il s'agit notamment d'économistes, d'entrepreneurs ou d'hommes politiques favorables, voire impliqués dans les réformes économiques menées en Russie à partir de 1992 par le gouvernement Gaïdar. Ces réformes ont été conçues par une équipe d'économistes néo-libéraux à la fois russes et occidentaux ${ }^{98}$. Ces derniers tiennent en conséquence un discours extrêmement proche de ceux qui pouvaient être formulés au début des années 1990 par les réformateurs russes. Selon Anders Aslund ${ }^{99}$, les causes du problème criminel russe doivent être cherchées dans l'héritage communiste. Pour que la criminalité diminue, « une libéralisation beaucoup plus radicale est avant tout nécessaire $»^{100}$. Les relations entre criminalité et économie sont certes indéniables et inquiétantes, mais d'une part temporaires, d'autre part secondaires si l'on considère que les mesures de libéralisation et de privatisation ont rendu irréversible un retour à l'ordre ancien ${ }^{101}$. Des comparaisons entre le contexte russe du début des années 1990 et le développement du capitalisme dans d'autres zones géographiques (notamment le rôle des robber barons dans l'industrialisation américaine au XIXème siècle) peuvent être menées. D'une certaine manière, les organisations criminelles peuvent même être considérées comme des acteurs contribuant à la mise en œuvre des réformes néo-libérales. Le marché est le seul mode de régulation susceptible d'éliminer les causes de la criminalité en Russie et de marginaliser le recours à des pratiques économiques illicites ${ }^{102}$. La libéralisation de l'économie provoquera à terme la diminution de l'économie de l'ombre, qui est la réponse normale des populations à une régulation répressive de l'activité économique ${ }^{103}$. 
Consacrant un article aux représentations du crime organisé russe dans le monde occidental, Phil Williams oppose ce qu'il appelle des évaluations "optimistes » et «pessimistes ». Il reproche aux premières d'accorder trop d'importance aux activités économiques du crime organisé, au rôle éventuellement positif que celui-ci peut jouer dans la transition économique et de sous-estimer sa capacité de maintenir les conditions qui lui ont permis de se développer. Selon lui, les évaluations "pessimistes", moins analytiques, conduisent en revanche à des exagérations et à des simplifications. Il appelle à un examen précis des sources disponibles sur le crime organisé russe et sur son expansion à l'étranger, notamment aux Etats-Unis ${ }^{104}$.

Or, les faits relatifs à la pénétration du crime organisé russe aux Etats-Unis sont rares. Au milieu des années 1990, les services répressifs commandent des rapports d'activité ou des recherches sur ce thème. Les services de lutte contre le crime organisé de trois Etats américains ${ }^{105}$ réalisent par exemple un rapport destiné à comprendre la criminalité commise par des Russes et à «assister les agences répressives dans leur effort croissant pour combattre la menace du crime organisé ${ }^{106}$. Ce rapport est basé sur les 404 dossiers dont disposent les trois agences commanditaires, ainsi que sur des sources orales. Il conclut qu'un milieu criminel russe est en activité dans les trois Etats considérés (New Jersey, New York, Pennsylvanie), mais qu'il est désorganisé. Il s'agit bien souvent de petites bandes réunies par la perspective d'une opportunité criminelle. Les relations entre criminels ne sont pas caractérisées par des liens hiérarchiques, mais par la méfiance. Dans quelques cas, des ententes entre des criminels russes et des organisations criminelles italiennes (Cosa Nostra) ou colombiennes ont pu être établies. Bien souvent en revanche, les infractions observées sont commises au sein de la communauté émigrée, au détriment des Russes les plus récemment arrivés (escroqueries, extorsions), ce qui est banal. Une recrudescence des homicides est observée depuis 1991: 65 cas ont été enregistrés entre 1981 et 1996, dont 40 après 1991. Les pratiques de blanchiment semblent répandues, mais dépassent le cadre de l'enquête, dans la mesure où ces opérations n'impliquent pas nécessairement la présence physique de criminels sur le sol américain. D'autres rapports d'institutions américaines impliquées dans la lutte contre la criminalité aboutissent aux mêmes conclusions. En Californie, des affaires d'extorsion, d'escroquerie ou de fraude liées au commerce du carburant, de fraude aux assurances, aux télécommunications, de vol de véhicules et de trafic de stupéfiants (quatre affaires entre 1992 et 1995), liées à des ressortissants des anciennes républiques soviétiques, ont été enregistrées ${ }^{107}$. Les douanes américaines font état de 82 affaires (dont deux affaires de stupéfiants), closes ou non, liées à des Russes et enregistrées entre 1991 et 1996. Le développement consacré par cette administration au danger que représente l'accès de la mafia russe aux matières nucléaires n'aboutit pas à la présentation de cas concrets ${ }^{108}$.

Les analogies entre les modes de constitution du problème étudié, en Russie et aux Etats-Unis, sont frappantes. Les formulations concurrentes du problème criminel sont relayées dans le monde occidental et mobilisées pour défendre des intérêts parfois spécifiques. La formation de coalitions d'intérêts contribue à la victoire, au moins temporaire, de la formulation la plus alarmiste des dangers que cause ou causera la mafia russe au monde occidental. Le contexte post-bipolaire permet de comprendre la genèse et le succès de cette formulation, fondée sur différents types de discours et dont les enjeux concernent autant le mode de développement souhaité pour la Russie que les relations entretenues par le monde occidental avec ce pays. L'enjeu d'une 
différenciation des discours scientifiques sur la mafia russe est donc majeur, car susceptible de contribuer à une formulation moins émotive du problème considéré.

\section{La lente différenciation d'un savoir scientifique}

Depuis le milieu des années 1990, une tendance à la différenciation des discours scientifiques sur le crime organisé russe s'observe, même si la grande majorité des publications s'inscrit dans la continuité des approches décrites précédemment.

Cette différenciation s'est opérée de deux manières, à partir d'emprunts aux travaux scientifiques menés sur le crime organisé dans d'autres contextes et à partir d'approches originales.

Dans le premier cas, le paradigme de la mafia italienne est incontournable. De même que la mafia représente la référence fondamentale des organisations criminelles, du fait de son histoire et de sa structuration, de même les travaux scientifiques qui lui sont consacrés balisent la connaissance sur le crime organisé en général. Au cours des années 1990, des grilles d'analyse élaborées pour comprendre la mafia italienne sont exportées dans le contexte russe.

Ainsi, les analyses de l'économiste Diego Gambetta sur l'industrie de la protection et la mafia sicilienne ${ }^{109}$ ont pu être mobilisées pour comprendre le contexte russe. Federico Varese explique la place du crime organisé en Russie par des défaillances institutionnelles. "Certains aspects des droits de propriété sont faiblement définis, un système fiscal prédateur empêche les propriétaires d'extraire légalement un revenu des biens qu'ils possèdent, et de solides doutes demeurent quant à la capacité de l'Etat de lutter efficacement contre la criminalité et la corruption $»^{110}$. En conséquence, le contournement de ces difficultés préoccupe les entrepreneurs, qui cherchent par exemple des moyens non étatiques de défendre leur propriété. Le climat de faible confiance vis-à-vis de l'Etat entraîne une demande de sources alternatives de protection, ce qui fournit des éléments de comparaison entre la Russie et la Sicile du XIXème siècle ${ }^{111}$. Pour d'autres auteurs, la protection assurée par les organisations criminelles est un "partenariat imposé » (silovoe partnerstvo). Dans la continuité des travaux classiques de Diego Gambetta et de Pino Arlacchi - qui placent l'accumulation $\mathrm{du}$ capital au centre des préoccupations de la mafia entrepreneuriale ${ }^{112}$ - Vadim Volkov considère que les organisations criminelles russes sont des "entreprises de violence ", démontrant l'incapacité de l'Etat à conserver le monopole de la violence légitime ${ }^{113}$.

La mobilisation du paradigme italien dans les analyses du crime organisé se heurte à des difficultés. Le caractère parcellaire et parfois biaisé des approches proposées par Pino Arlacchi ou Diego Gambetta a déjà été relevé. Pour Salvatore Lupo, l'opposition effectuée par Pino Arlacchi entre mafias traditionnelle et moderne n'est pas convaincante. Selon l'historien, le caractère entrepreneurial existe depuis longtemps, mais fut souvent occulté par le «tableau volontairement minimaliste que le mafieux fait de lui-même $»^{114}$. Par ailleurs, cette entreprise n'est hier comme aujourd'hui guère innovante, et s'apparente davantage à la gestion d'une rente. Quant à Gambetta, il "sous-évalue le facteur extorsion par rapport au facteur protection. La mafia d'ordre présuppose toujours un désordre à organiser et à contrôler, dans la Sicile post-unitaire comme au cours des plus récentes escalades de la délinquance, et c'est donc, dans une large mesure, la mafia qui crée l'insécurité dont elle profite, au point que son unique fonction est, peut-on dire, celle qu'elle détermine elle-même $»^{115}$. 
Les critiques adressées aux approches proposées par P. Arlacchi ou D. Gambetta doivent cependant être tempérées par leur apport historique indéniable à la connaissance de la mafia italienne. Comme l'a noté Jean-Louis Briquet, la mafia, en évoluant constamment, a causé une adaptation correspondante des grilles d'analyse et des approches scientifiques développées pour la saisir ${ }^{116}$. Cette remarque fait cependant surgir une nouvelle difficulté, compliquant encore la comparaison entre contextes italien et russe. L'historicité des pratiques mafieuses en Russie est différente puisque les organisations criminelles les mieux structurées sont nées dans l'Union Soviétique des années 1970. Par ailleurs, les bouleversements politiques et économiques apparus à partir de la fin des années 1980 ont entraîné l'émergence simultanée de nombreuses pratiques: diversification des activités, légales et illégales, activités entrepreneuriales, industrie de la protection, évergétisme, formes de redistribution sociale au niveau local, usage immodéré de la violence et de la corruption, échanges de services entre le milieu criminel et le milieu politique.

Les chercheurs russes apportent par ailleurs aujourd'hui des outils d'analyse et des connaissances susceptibles d'éclairer les recherches générales sur le crime organisé. Les auteurs des articles présentés dans ce numéro développent des approches qui participent à la constitution d'un savoir scientifique différencié. Ces différents travaux, parmi d'autres ${ }^{117}$, montrent que la recherche scientifique russe sur le crime organisé a atteint une nouvelle étape: il ne s'agit plus de s'interroger sur l'existence de ce phénomène ou de l'expliquer en proposant une interprétation fondée sur des présupposés idéologiques, mais de proposer des approches nouvelles susceptibles de consolider les connaissances et in fine d'être exportées dans d'autres contextes géographiques.

\section{NOTES}

1. Naylor R. T. , «From cold war to crime war : the search for a new 'national security threat' », Transnational organized crime, vol.1, $\mathrm{n}^{\circ} 4$, hiver 1995, pp.37-56.

2. Edelman M, Pièces et règles du jeu politique, Paris, Le Seuil, 1991, p.42 (Traduction de: Constructing the political spectacle, Chicago, Chicago University Press, 1988).

3. Ibid., p.43.

4. Ibid., p.14. Pour une illustration de la pertinence de cette approche appliquée au cas soviétique, voir Verdier E., " Mafia et politique en U.R.S.S. », Cultures \& Conflits, n $^{\circ}$ 3, automne 1991, pp.55-74.

5. Comme le considère Fernando Acosta, le crime ne peut être analysé que si trois dimensions sont considérées : factuelle, axiologique et normative« [...] Le crime n'est pas exclusivement un acte, ni même exclusivement un acte blâmable, mais le rapport entre une manière de faire et une manière de définir-agir-résoudre une situation problématique. Nous avons donc d'un côté un fait brut et de l'autre un fait mental accompagné d'une pratique institutionnelle. C'est de cette combinaison d'éléments qu'il faut rendre compte ", Acosta F., "Constructivisme versus réalisme. Quelques réflexions sur les notions de crime, déviance et situations problématiques », in 
Cartuyvels Yet al., Politique, police et justice au bord du futur, Paris, L'Harmattan, 1998, pp.40-41. $C^{\prime}$ est l'auteur qui souligne.

6. Je ne vais pas présenter dans le cadre de cet article l'histoire de l'expression « criminalité organisée » dans le monde occidental, sa fragilité sémantique, les critiques adressées à cette notion et aux présupposés qui la fondent. Sur ce sujet, voir notamment: Reuter P., Disorganized crime, Cambridge, MIT Press, 1983 ; Bigo D., "Pertinence et limites de la notion de crime organisé », Relations internationales et stratégiques, n²0, 1995, pp.134-138.

7. J'ai eu l'occasion d'analyser l'évolution du dispositif répressif (législation pénale, restructuration des services de lutte, mise en œuvre des décisions politiques) en Russie soviétique et post-soviétique. Voir Favarel-Garrigues G., La lutte contre la criminalité économique en Russie soviétique et post-soviétique (1965-1995), Thèse de doctorat, soutenue en décembre 2000 à l'Institut d'Etudes Politiques de Paris.

8. «Nous anéantissons l'antagonisme entre l'individu pris isolément et tous les autres, nous opposons la paix sociale à la guerre sociale, nous coupons la racine même du crime et, ce faisant, nous rendons superflue une bonne partie de l'activité actuelle des organes judiciaires et administratifs », Engels F., « Elberfeldskie retchi » (Les discours d'Elberfeld), in Marx K., Engels F., Sotchinenia, Izdanie vtoroe, Tom 2 (๕uvres, Seconde édition, Tome 2), Moscou, Gosoudarstvennoe izdatel'stvo polititcheskoï literatoury, 1955, pp.537-538. Le terme "racine » est souligné dans l'édition consultée.

9. Bolchaïa Sovetskaïa Entsiklopedia, Tom 20 (La Grande Encyclopédie Soviétique, Tome 20), Moscou, Izdatelstvo « Sovetskaïa Entsiklopedia », 1975, p.539.

10. Sur la criminalité professionnelle, voir le premier ouvrage d'Alexandre Gourov, plus tard à la tête de la direction centrale de la lutte contre la criminalité organisée, au ministère de l'Intérieur. Gourov A., Professionalnaïa prestoupnost (La criminalité professionnelle), Moscou, Iouriditcheskaïa literatoura, 1990.

11. Il semblerait en revanche que des recherches aient été menées dès les années 1970 sur l'économie souterraine, à partir d'études économiques sur la consommation des ménages ou sur le travail au noir. Voir Koriagina T., «Analiz, otsenki, prognozy» (Analyse, évaluations, prévisions), in Collectif, Tenevaïa ekonomika (L'économie souterraine), Moscou, Ekonomika, 1991, pp. 28-30. Les premiers ouvrages accessibles sur l'économie souterraine datent de 1987 : Nikolaeva M., Seviakov A., Tenevaïa ekonomika: metody analiza i otsenki (L'économie souterraine: méthodes d'analyse et d'évaluation), Moscou, TsEMI AN SSSR, 1987.

12. Intervention de O. V. Pristanskaïa in Organizovannaïa prestoupnost' (La criminalité organisée), Moscou, Iouriditcheskaïa literatoura, 1989, p.101.

13. Par exemple, les films Briliantovaïa rouka (Le bras de diamant) ou Djentelmeny oudatchi (Les gentlemen de la réussite).

14. Voir notamment l'ouvrage que l'auteur des Récits de la Kolyma, incarcéré jusqu'en 1953, a consacré à ce sujet : Chalamov V., Essais sur le monde du crime, Paris, Gallimard, 1993.

15. Voir par exemple l'ouvrage "Ougolovnaïa Rossia» (La Russie criminelle) écrit en 1975 par Valeri Tchalidze, publié à l'étranger et contenant en annexe un glossaire du langage de la pègre, daté de 1964 et destiné à l'usage des agents de la police judiciaire de la ville de Kiev. En français : Chalidze V., Le crime en Union soviétique, Paris, Olivier Orban, 1978.

16. Voir Favarel-Garrigues, G., op. cit. (chapitre II).

17. Sur ces affaires bien connues, voir Vaksberg A., La mafia russe, Paris, Albin Michel, 1992.

18. Voir notamment Gleason G., "Nationalism or organized crime? The case of the 'cotton scandal' in the USSR ", Corruption and reform, $n^{\circ}$ 5, 1990, pp.87-108.

19. Pour une récente contribution à la connaissance de la mafia du commerce, voir Duhamel L., "Justice and politics in Moscow 1983-1986 : the Ambartsumyan case », Europe-Asia Studies, vol. $52, \mathrm{n}^{\circ}$ 7, 2000, pp.1307-1329.

20. Favarel-Garrigues G., op. cit., pp.175-176. 
21. Sur ces points, voir Favarel-Garrigues G., «Privatisation et changement politique en Russie soviétique et post-soviétique » in Hibou B. (dir.), La privatisation des Etats, Paris, Karthala, 1999, pp.247-284, et « Eléments pour une compréhension de l'évolution des délinquances économiques et financières en Russie », Les Cahiers de la sécurité intérieure, n 36, deuxième trimestre 1999, pp.177-186.

22. Voir Clement K., «La pauvreté de masse en Russie », Cultures \& Conflits, $n^{\circ} 35$, automne 1999, pp.35-70.

23. Gourov A., Krasnaïa mafia (La mafia rouge), Moscou, Samotsvet, Miko «Kommertcheskii vestnik», 1995, pp.28-29.

24. «600 secondes exploite une formule simple mais efficace: dépister la face cachée de la société soviétique. Un sujet révèle par exemple que l'ex-premier secrétaire du Parti de Leningrad a utilisé ses contacts pour acheter une limousine Mercedes à un prix défiant toute concurrence ; un autre démasque une usine de viande fabriquant des saucisses avec de vieilles carcasses; un troisième décrit avec force détails sordides comment des sprays insecticides sont délabellisés et vendus dans des magasins d'Etat en lieu et place de déodorants ", Nivat A., Quand les médias russes ont pris la parole, Paris, L'Harmattan, 1997, p.263.

25. Briquet J. L. , «Comprendre la mafia. L'analyse de la mafia dans l'histoire et les sciences sociales ", Politix, n 30, 1995, p.139.

26. Ibid., p.139.

27. Levada Y., Entre le passé et l'avenir. L'homme soviétique ordinaire, Paris, Presses de la FNSP, 1993, p.41 (Plusieurs réponses peuvent être données par chaque personne interrogée).

28. Queloz N., «A-t-on encore des raisons de distinguer criminalités économique et organisée ? ", Les Cahiers de la sécurité intérieure, n 36, deuxième trimestre 1999, pp.21-40.

29. Traduction littérale de l'expression « tenevaïa ekonomika ».

30. Oulybin KA., "Znakomaïa neznakomka » (Une inconnue familière), in Tenevaïa ekonomika (L'économie de l'ombre), Moscou, Ekonomika, 1991, p.7.

31. Docteur en droit, Alexandre Gourov deviendra en 1989 le premier directeur des services de lutte contre le crime organisé au sein du ministère de l'Intérieur de l'U.R.S.S. Il est l'auteur de nombreux ouvrages sur la pègre soviétique et post-soviétique.

32. Le mois suivant, la Literatournaïa Gazeta publie un nouvel article sur la criminalité organisée (Sokolov V., «Bandokratiâ » (La bandocratie), Literatournaïa Gazeta, 17 août 1988). Suite à ces articles, la rédaction de l'hebdomadaire reçoit un courrier abondant, dont il est notamment rendu compte dans l'édition du 28 septembre 1988 («Pryjok lva na glazakh izoumlennoï poubliki » (Le saut du lion sous les yeux du public ahuri), Literatournaïa Gazeta, 28 septembre 1988).

33. Alexandre Gourov reproduit, dans l'un de ses ouvrages, les réactions officielles à l'entretien qu'il a accordé à la Literatournaïa Gazeta, notamment une note émanant du Comité Central du P.C.U.SVoir Gourov A., Krasnaïa mafia, op. cit. , pp.19-27.

34. Literatournaïa Gazeta, 28 septembre 1988.

35. La prokouratoura, chargée de veiller au respect de la légalité sur tout le territoire et dans tous les domaines du droit, possède, comme le ministère de l'Intérieur ou les services de sécurité de l'Etat, ses propres organes d'instruction.

36. Ivanov L., Ilina L., Pouti i soudby otetchestvennoï kriminologii (Voies et destin de la criminologie patriotique), Moscou, Naouka, 1991; Solomon Jr PH., Soviet criminologists and criminal policy : specialists in policymaking, New York, Columbia University Press, 1978.

37. La criminalité organisée fait l'objet d'articles dans les grandes revues juridiques à partir de 1988. Cf. Sovetskoe Gosoudarstvo i Pravo, $n^{\circ} 5,1988, n^{\circ} 7,1989$; Sotsialistitcheskaïa Zakonnost, $n^{\circ} 6,1988, n^{\circ} 9,1988, n^{\circ} 4,1989, n^{\circ} 6,1989$. 
38. Ils sont également les principaux membres de l'Association criminologique, qui édite depuis la fin des années 1980 des ouvrages très fréquemment cités, en Russie ou dans le monde occidental.

39. Sur ces points, voir la thèse que j'ai consacrée à « la lutte contre la criminalité économique en Russie soviétique et post-soviétique, de 1965 à 1995 », op. cit.

40. Sommier I., Les mafias, Paris, Montchrestien, 1998, pp.61-64.

41. Hormis quelques exceptions telles que: Gorianovski A., "Oukrast' mojno vsioRezoultaty vklioutchennovo nablioudenia » (On peut tout voler. Résultats d'une observation participante), Sotsiologitcheskie Issledovania, $n^{\circ} 2,1990$, pp.56-64.

42. Sur Moscou: Soldatenko B., Moskva kriminalnaïa (Moscou la criminelle), Moscou, Tsentrpoligraf, 2000; Sur Saint-Petersbourg: Konstantinov A., Banditski Peterbourg (Le Petersbourg du crime), Saint-Petersbourg, Folio-Press, 1997. Sur Ekaterinbourg, voir la revue "Sychtchik» (Le limier), exclusivement consacrée au crime organisé dans cette ville et dans l'Oural en général.

43. Edelman M., op. cit., p.47.

44. Favarel-Garrigues G., op. cit. (deuxième partie).

45. "Je soutiens que $90 \%$ des entrepreneurs actuels sont des voyous ", Govoroukhine S., Strana vorov na doroge $v$ svetloe boudouchtchee (Le pays des voleurs en route vers l'avenir radieux), Narva, Firma «Chans ", 1994, p.164. Cinéaste reconnu, Stanislas Govoroukhine a réalisé à partir de la fin des années 1980 des documentaires censés refléter l'état de la Russie. Les deux premiers («Impossible de vivre ainsi », 1989 ; «La Russie que nous avons perdue », 1992) ont connu un succès extraordinaire. Stanislas Govoroukhine est par la suite devenu député, mais l'impact de ses documentaires a progressivement diminué.

46. Il s'agit de la traduction française de l'ouvrage précédemment cité de Stanislas Govoroukhine (Lausanne, L'âge d'homme, 1995).

47. Govoroukhine S., op. cit.

48. Ibid., p.43.

49. Discours prononcé par A. Routskoï, Président de la commission interdépartementale de la lutte contre la criminalité et la corruption, au cours d'une réunion tenue à Moscou le 12 février 1993, in : Neizvestny Routskoï : polititcheski portret (Routskoï, cet inconnu : portrait politique), Moscou, Obozrevatel', 1994, p.211.

50. «Les partisans d'une ' privatisation-à-la-Tchoubaïs' créent les conditions de formation d'un immense capital compradore et, avec la légèreté des cosmopolites, sont prêts à soumettre le pays à des intérêts étrangers ", in Neizvestny Routskoï : polititcheski portret (Routskoï, cet inconnu : portrait politique), Moscou, Obozrevatel', 1994, p445Même constat chez l'économiste Tatiana Koriagina : les réformes économiques menées par Egor Gaïdar et son équipe sont « un diktat de l'Occident et du milieu criminel » (Rabotchaïa Tribouna, 7 février 1992).

51. Govoroukhine S., op. cit., pp.33-34, p.84.

52. Iarotchkin V., Organizovannaïa prestoupnost'Otkouda iskhodit ougroza (La criminalité organisée. D'où vient la menace), Moscou, « Os'-89 », 1995, pp.3-4.

53. Organizovannaïa prestoupnost - 3 (La criminalité organisée - 3), Moscou, Kriminologitcheskaïa Assotsiatsia, 1996, p.120.

54. Govoroukhine S., op. cit., pp.303-304 et 355-356.

55. Ce qui n'empêche pas les connivences. Voir Shelley L., «Is the Russian State coping with organized crime and corruption?", in Sperling V. (dir.)., Building the Russian StateInstitutional crisis and the quest for democratic governance, Boulder, Westview, 2000, pp.105-106.

56. Ces points sont développés dans ma thèse, pp.449-450.

57. En langue française, voir par exemple l'entretien avec Alexandre Lebed, intitulé «Russie: tant que le crime règnera... » in Politique internationale, nº 84, été 1999, pp.243-256. 
58. Voir par exemple : Aslakhanov A., O mafii v Rossi bez sensatsiiRazmychlenia general-maïora militsii (Sur la mafia en Russie sans sensationnalismeRéflexions d'un général de brigade de police), Moscou, Institout massovykh kommounikatsii, 1996.

59. En Russie, on appelle "privatisations spontanées » les transferts de propriété qui se sont effectués avant 1992, alors qu'aucun cadre général ne les réglementait.

60. Tchoubaïs A. (dir.), Privatizatsia po-rossiski (La privatisation à la russe), Moscou, Vagrious, 1999, p.358.

61. "L'URSS n'avait jamais connu programme de réformes aussi concret et radical. Le terme de socialisme n'y était jamais employé. Ce texte peut être considéré comme la percée la plus importante de la pensée économique non socialiste en Union soviétique ", Aslund AGorbachev's struggle for economic reform, Ithaca, Cornell University Press, 1991, p.209.

62. Perekhod k rynkouKontseptsia i programma (La transition vers le marchéConception et programme), Moscou, Arkhangelskoe, 1990, p.135.

63. Ibid., p.136.

64. Ibid., p;139. Ce chiffre, considéré comme un élément de propagande, a été vivement contestéVoir sur ce point : Kolesnikov V., Ekonomitcheskaïa prestoupnost i rynotchnye reformy (La criminalité économique et les réformes marchandes), Saint-Petersbourg, Izdatel'stvo SanktPeterbourgskovo ouniversiteta ekonomiki i finansov, 1994, p.106.

65. Osipenko O., Svoe delo (Sa propre affaire), Moscou, Politizdat, 1991, pp.78-88.

66. Ispravnikov V., «Tenevaïa ekonomika : ekspropriatsia, otmyvanie ili legalizatsia?» (L'économie de l'ombre: expropriation, blanchiment ou légalisation?), in Tenevaïa ekonomikaEkonomitcheskie, sotsialnye i pravovye aspekty (L'économie de l'ombre. Aspects économiques, sociaux et juridiques), Moscou, Nezavisimy blagotvoritelny fond koultoury «Foros », 1996, p.11.

67. Kommersant, 17 avril 1994.

68. Déclaration effectuée à la télévision, NTV, 6 mai 1997Sur la position d'Edouard Rossel vis-àvis de la criminalité économique, voir également: Frisby T., «The rise of organised crime in Russia : its roots and social significance », Europe-Asia Studies, vol50, n 1, 1998, p.46.

69. Nezavisimaïa Gazeta, 20 avril 1999.

70. D'autres exemples d'emploi de ce discours par des personnalités politiques, par exemple le gouverneur de la région de Novgorod, figurent dans une récente étude émanant de Global Programme against Money Laundering, Office for Drug Control and Crime PreventionVoir Solongo D., Russian Capitalism and Money-Laundering, New York, United Nations, 2001, pp.15-17. 71. Ovtchinski V., Eminov, VIablokov, N. (dir.), Osnovy borby s organizovannoï prestoupnostiou (Fondements de la lutte contre la criminalité organisée), Moscou, Infra-M, 1996, p.172. Voir également les violentes critiques du Programme des 500 jours in: Organizovannaïa prestoupnost' - 2 ( La criminalité organisée - 2), Moscou, Kriminologitcheskaïa Assotsiatsia, 1993, pp169-170; Organizovannaïa prestoupnost' - 3 (La criminalité organisée - 3), Moscou, Kriminologitcheskaïa Assotsiatsia, 1996, pp.53-55.

72. Iourev L., "Mesto v teniDialog s gospodinom N. » (Une place à l'ombre. Dialogue avec Monsieur N.), Biznes, $n^{\circ} 3,1991$, p.4.

73. Interview d'un racketteur in: Ogoniok, $n^{\circ} 30,1991$. Ces discours sont également présents dans les entretiens réalisés par Iakov Gilinski et Iakov Kostioukovski. Voir leur contribution dans ce numéro.

74. Golos Osoujdiennykh khoziaïstvennikov, $n^{\circ} 1$, novembre 1990, p.34.

75. Organizovannaïa prestoupnost' - 2 (La criminalité organisée - 2), Moscou, Kriminologitcheskaïa assotsiatsia, 1993, p170.

76. Inspiré par le sous-titre « Russians are coming! » in Naylor RT., «From cold war to crime war : the search for a new 'national security' threat ", Transnational organized crime, vol.1, $\mathrm{n}^{\circ} 4$, hiver 1995, pp.37-56. 
77. Nadelman E., Cops across borders: the internationalization of US criminal law enforcementUniversity Park, The Pennsylvania State University Press, 1993 ; Friman H. R., Narcodiplomacy : exporting the US war on drugs, Ithaca, Cornell University Press, 1996.

78. Handelman S., Comrade criminal, Londres, Michael Joseph, 1994.

79. Lesnik R., Blanc H., L'Empire de toutes les mafias, Paris, Presses de la Cité, 1996.

80. Sur tous ces points, voir par exemple les chapitres "Les loups des steppes de l'Est » et "L'Armée Rouge, berceau des tueurs » in Ziegler J., Les seigneurs du crime. Les nouvelles mafias contre la démocratie, Paris, Le Seuil, 1998, pp.81-174.

81. Sterling C., The terror network: the secret war of international terrorism, New York, Reader's Digest Press, 1981.

82. Sterling C., Thieves' world : the new global threat of organized crime, New York, Simon and Schuster, 1995.

83. Pour une stimulante présentation critique des thèses de Claire Sterling, voir Naylor R. T., op. cit., pp.37-56. R. T. Naylor cite notamment des propos de responsables politiques ou de dirigeants des services répressifs américains selon lesquels « le crime organisé est le nouveau communisme, la nouvelle menace monolithique » ou « avoir gagné la guerre froide ne représente rien alors que nous sommes en passe de perdre la guerre contre une autre tyrannie» (p.37).

84. Voir par exemple le rapport: Raine L., Cilluffo F. (dir.), Global organized crime. The new empire of evil, Washington, DC., Center for Strategic and International Studies, 1994.

85. Cilluffo F., Burke G. (dir.), Russian organized crime, Washington, DC., Center for Strategic and International Studies, 1997.

86. Cilluffo F., Burke G., op. cit., p.2.

87. Ziegler J., op. cit., p.109.

88. Voronin Y., «The emerging criminal State : economic and political aspects of organized crime in Russia », Transnational organized crime, vol.2, n 2-3, été-automne 1996, p.56.

89. Castells M., Fin de millénaire, Paris, Fayard, 1999, p.208.

90. Ibid., p.195.

91. Ibid.

92. Ziegler J., Les seigneurs du crime. Les nouvelles mafias contre la démocratie, Paris, Le Seuil, 1998, p. 13.

93. Einstein S., Amir M. (dir.), Organized crime: uncertainties and dilemmas, Chicago, Office of International Criminal Justice, 1999, p.90.

94. Sommier I., "Violence politique et violence criminelle: la porosité des frontières", in Crettiez X., Ferret J. (dir.), Le silence des armes? L'Europe à l'épreuve des séparatismes violents, Paris, La documentation française, 1999, pp33-50.

95. Il serait d'ailleurs intéressant de montrer précisément comment la justification des deux guerres tchétchènes associe les registres anti-terroriste et anti-mafieux.

96. Einstein S., Amir M. (dir.), op. cit., p.87.

97. Ibid., p.88.

98. Sur la constitution de cette équipe, voir: Wedel J., «Rigging the U.S.-Russian relationshipHarvard, Chubais and the transidentity game ", Demokratizatsya, vol.7, $\mathrm{n}^{\circ} 4$, automne 1999, pp.469-500.

99. Anders Aslund fut l'un des conseillers occidentaux d'Egor Gaïdar lors de la mise en œuvre des réformes de 1992.

100. Aslund A., How Russia became a market economy, Washington, DC., The Brookings Institution, 1995, p.170. Les économistes impliqués dans la conception et la mise en œuvre des réformes de 1992 ont très rapidement considéré que la politique qu'ils prônaient n'avait pas été complètement menée. Selon eux, la thérapie de choc qu'ils appelaient de leurs vœux ne s'est jamais vraiment produite. 
101. La garantie de l'irréversibilité de la transition vers l'économie de marché était l'un des principaux arguments employés pour défendre le processus de privatisation en 1992.

102. Voir par exemple: Anderson A., "The red mafia : a legacy of communism », in Lazear E. (dir.), Economic transition in Eastern Europe and Russia : realities of reformStanford, The Hoover Institution Press, 1995 ; Litwack J., "Legality and market reform in Soviet-type economies ", Journal of economic perspectives, vol.5, $\mathrm{n}^{\circ} 4$, automne 1991, p.78.

103. Lotspeich R., «Crime in the transition economies ", Europe-Asia Studies, vol47, n 4, 1995, pp. 565-566. Voir également: Leitzel J., Russian economic reform, Londres, Routledge, 1995, pp. 43-45 ; Leitzel J., Gaddy C., Alexeev M., « Mafiosi and matrioshki : organized crime and Russian reform », Brookings Review, hiver 1995, pp.26-29.

104. Williams Ph., "Introduction: how serious a threat is Russian organized crime?", Transnational organized crime, vol.2, n 2-3, été-automne 1996, pp.3-11.

105. Les commanditaires sont: New York State Organized Crime Task Force, New York State Commission of Investigation, Pennsylvania Crime Commission, New Jersey State Commission of Investigation.

106. Finckenauer J., Waring E., « Russian Emigré Crime in the United States : Organized Crime or Crime that is Organized?», Transnational organized crime, vol.2, n 2-3, été-automne 1996, pp. 139-140.

107. "'Russian organized crime: California's newest threat', report by Daniel ELungren, Attorney General, California Department of Justice, March 1996 », Trends in organized crime, vol. 2, n 1, automne 1996, pp.32-35.

108. «George JWeise, Commissionner, US Customs Service, Statement before Committee on Governmental Affairs, Senate Permanent Subcommittee on Investigations, Hearings on Russian Organized Crime in the United States, 15 May 1996 ", Trends in organized crime, vol.2, nº 1 , automne 1996, pp.8-12.

109. Gambetta D., The sicilian mafia : the business of private protection, Cambridge, Harvard University Press, 1993.

110. Varese F., "The transition to the market and corruption in post-socialist Russia ", Political Studies, vol.XLV, 1997, p.596.

111. Varese F., "Is Sicily the future of Russia? Private protection and the rise of the Russian mafia », Archives européennes de sociologie, vol.35, n², 1994, pp.224-258.

112. Arlacchi P., L'éthique mafiosa et l'esprit du capitalisme, Grenoble, Presses Universitaires, 1986.

113. Volkov V., "Violent entrepreneurship in post-communist Russia », Europe-Asia Studies, vol.51, n 5, 1999, pp.741-754 (traduit in : Politix, vol.13, n 49, 2000, pp57-75).

114. Lupo S., Histoire de la mafia des origines à nos jours, Paris, Flammarion, 1999, pp.27-28.

115. Ibid., pp.29-30.

116. Briquet J-L., op. cit., p139.

117. Voir notamment les entretiens réalisés par Igor Kliamkin et Lev Timofeev auprès de différentes catégories sociales afin de mieux comprendre la place des pratiques économiques illicites dans la société russe actuelleCfKliamkin I., Timofeev L., Tenevaïa Rossia (La Russie de l'ombre), Moscou, RGGOu, 2000. 
INDEX

Mots-clés : discours sécuritaire, mafias et crime organisé

Index géographique : Russie 